\title{
POINTWISE BOUNDED APPROXIMATION AND ANALYTIC CAPACITY OF OPEN SETS
}

\author{
BY
}

\author{
STEVEN JACOBSON
}

\begin{abstract}
We examine the semi-additivity question for analytic capacity by studying the relation between the capacities of bounded open sets and their closures.
\end{abstract}

Introduction. Definitions and basic properties of analytic capacity, along with its most important uses, can be found in [4] or [20]. Let $\mathbf{C}$ denote the complex plane, and $C^{*}$ the Riemann sphere. We write $\gamma(S)$ and $\alpha(S)$ for the analytic capacity and continuous analytic capacity of a set $S \subset \mathrm{C}$. The class of functions defining $\gamma(S)$ will be called admissible, and the class defining $\alpha(S)$ will be called $C$-admissible. If $f$ satisfies the defining conditions for admissibility, except possibly that $f(\infty) \neq 0$, then $f$ will be called "admissible" (and the quotation marks are part of the word). An Ahlfors function for a compact set $K$ is a function $f$ admissible for $K$, with $f^{\prime}(\infty)=(2 \pi i)^{-1} \int f(z) d z=\gamma(K)$, where the path of integration is any circle surrounding $K$. Such a function always exists, and is uniquely determined on the unbounded component of $\mathrm{C}^{*}-K$.

The word neighborhood will always mean an open set. We will always use the letter $D$ for an open disc; $D(z, \delta)$ will be the open disc with center $z$ and radius $\delta$; and if $r>0$, then $r D$ will be the open disc with the same center as $D$, and with radius equal to $r$ times the radius of $D$. The symbol $d m$ will refer to integration with respect to planar Lebesgue measure, and we will use a double integral symbol for such integration. In place of the differential operator $1 / 2(\partial / \partial x+i \partial / \partial y)=\partial / \partial \bar{z}$ we will use the notation of the gradient. When the symbol, grad $\phi$, appears in an integral, it is understood to mean (grad $\phi)(w)$, where $w$ is the variable of integration. If $K \subset \mathrm{C}$ is compact, then $C(K)$ is the Banach algebra of all continuous, complex valued functions on $K$, with the supremum norm. $R(K)$ is the closed subalgebra generated by all rational functions with poles off $K$. If $U$ is open, $H^{\infty}(U)$ is the Banach algebra of all bounded analytic functions on $U$, with the supremum norm.

It has been conjectured that capacity has the property of semi-additivity:

Presented to the Society, January 24, 1975 under the title Analytic capacity of open sets; received by the editors February 18, 1975.

AMS (MOS) subject classifications (1970). Primary 30A82; Secondary 46J15. 
Does there exist a constant $M$ with the property that $\alpha(K \cup L) \leqslant M(\alpha(K)+\alpha(L))$ and $\gamma(K \cup L) \leqslant M(\gamma(K)+\gamma(L))$ whenever $K$ and $L$ are compact disjoint sets? Semi-additivity is known to be true in certain special cases, and it is even true that $\gamma(K \cup L) \rightarrow \gamma(K)+\gamma(L)$ as the distance between $K$ and $L$ approaches infinity. See Satz 4 of [12]. Studying the semi-additivity question in detail in [3] , A. Davie has found a number of equivalent formulations. In his Theorem 5.11, Davie shows that semi-additivity of $\alpha$ is equivalent to that of $\gamma$, and also that both of these are equivalent to the following statement. If $E$ is compact and $\gamma(E)=0$, then $\gamma(E \cup F)=\gamma(F)$ for all sets $F$. We claim that it suffices here to take $F$ to be an open set.

Proposition. The relation $\gamma(E \cup F)=\gamma(F)$ is true for all sets $F$ and for all compact sets $E$ with $\gamma(E)=0$ iff the relation $\gamma(E \cup V)=\gamma(V)$ is true for all bounded open sets $V$ and all compact sets $E$ with $\gamma(E)=0$.

Proof. We begin by noting the fact that if $B$ and $C$ are compact sets, then $B-C$ is locally compact. Now suppose $\gamma(E \cup V)=\gamma(V)$ for some compact set $E$ and for all bounded open sets $V$. Let $F$ be any set, and let $K \subset E \cup F$ be compact. Then $K-E$ is locally compact, and also $K-E \subset F$. In $\S 1$ of [3], Davie defines a set function $\gamma^{*}$ by $\gamma^{*}(S)=\inf \{\gamma(U): U$ is open, $S \subset U\}$, and in Theorem 5.1 of the same paper, he shows that there is an absolute constant $A$ satisfying $\gamma^{*}(S) \leqslant A \gamma(S)$ whenever $S$ is locally compact. Therefore we can choose an open set $U$ with $K-E \subset U$ such that $\gamma(U) \leqslant A \gamma(K-E)+\epsilon$. If we intersect $U$ with an open disc $D$ which contains $K$, then we get a bounded open set $V$ such that $K-E \subset V$ and $\gamma(V) \leqslant \gamma(U) \leqslant A \gamma(K-E)+\epsilon$. Therefore $K \subset E \cup V$, and $\gamma(K) \leqslant \gamma(E \cup V)=\gamma(V) \leqslant A \gamma(K-E)+\epsilon \leqslant A \gamma(F)+\epsilon$. Since $\epsilon$ and $K$ are arbitrary, $\gamma(E \cup F) \leqslant A \gamma(F)$, and then $\alpha(E \cup F) \leqslant A \gamma(F)$.

The conclusion we have established is part two of Theorem 5.11 of [3]. As that is equivalent to part three, we have proved half of our proposition. The other half is trivial.

In attempting to prove a statement like $\gamma(E \cup V)=\gamma(V)$ we see that the only difficulty is with the portion of $E$ which belongs to $\partial V$. Compact sets of zero analytic capacity are sets of removable singularities for bounded analytic functions. A function admissible for a compact subset $K$ of $E \cup V$ will therefore extend analytically across every point of $K-\bar{V}$ to become admissible for $K \cap \bar{V} \subset V \cup(E \cap \partial V)$. To prove $\gamma(V)=\gamma(E \cup V)$ it thus suffices to prove $\gamma(V)=\gamma(V \cup(E \cap \partial V))$. Note that $E \cap \partial V$ is a compact set of zero analytic capacity. (Incidentally, the remarks of this paragraph show that $\gamma(K \cup L)=$ $\gamma(L)$ whenever $K$ and $L$ are compact and $\gamma(K)=0$.)

This leads to the question of whether or not it is possible to add part of $\partial V$ on to $V$ without changing the analytic capacity. We plan to show that in many cases all of $\partial V$ can be added on, thus yielding the relation $\gamma(V)=\gamma(\bar{V})$. 
In the general case, a certain portion of $\partial V$ can always be added on. Finally we will show that the relation $\gamma(V)=\gamma(\bar{V})$ being true for a certain class of bounded open sets is equivalent to the semi-additivity of analytic capacity.

This paper is based on the author's doctoral dissertation, which has the same title and was written at Yale University. The author would like to thank Shizuo Kakutani, and Richard Basener, Theodore Gamelin, Thomas Gregory, Stuart Sidney, and Andrew Sommese for their valuable assistance and encouragement.

1. The main theorem. Our principal result about pointwise bounded approximation is Theorem 1 below. This is very similar to Vitushkin's Theorem and a large portion of the proof is the same. One way in which it differs is that we need the following difficult result of T. Gamelin and J. Garnett. This is the special case of Theorem 6.7 of [6] in which $P$ is taken to be the empty set.

REDUCTION OF NORM THEOREM. Let $U \subset \mathrm{C}^{*}$ be open and let $Q \subset \partial U$ be compact. Assume $R(Q)=C(Q)$. Let $J$ be a uniformly closed subalgebra of $H^{\infty}(U)$. Suppose that for each $F \in J$, there is a uniformly bounded sequence $\left\{F_{n}\right\}_{n=1}^{\infty}$ in $H^{\infty}(U)$ such that each $F_{n}$ extends to be analytic in a neighborhood of $Q$, and such that $\left\{F_{n}\right\}$ converges uniformly to $F$ on each subset of $U$ at positive distance from $Q$. Then there is a sequence in $H^{\infty}(U)$ having all these properties of the $F_{n}$, and in addition satisfying $\left\|F_{n}\right\| \leqslant\|F\|$.

COROLlary. The conclusion of the Reduction of Norm Theorem remains valid if we replace the hypothesis " $Q \subset \partial U$ " by " $Q \subset \mathrm{C}-U$."

It does not appear that this corollary follows from the statement of the Reduction of Norm Theorem. However the corollary does follow from the proof of that theorem if some minor changes are made. As a matter of fact, it is this modification of the Reduction of Norm Theorem, and not the theorem itself, which is used in the proof of Theorem 6.9 of [6].

THEOREM 1. Let $W \subset \mathrm{C}^{*}$ be an open set containing the point $\infty$. Let $E \subset \mathrm{C}-W$ be compact, with $R(E)=C(E)$. Then the first four statements below are equivalent, and each of them implies the fifth one. In the special case that $E$ has Lebesgue measure zero, all the five statements are equivalent.

(a) $\gamma(G-W)=\gamma(G-(W \cup E))$ for every bounded open set $G$.

(b) $\gamma(D-W)=\gamma(D-(W \cup E))$ for every open disc $D$.

(c) For each $z \in E \cap \partial W$, there exists $r=r(z) \geqslant 1$ such that

$$
\liminf _{\delta \rightarrow 0} \frac{\gamma(D(z, r \delta)-(W \cup E))}{\gamma(D(z, \delta)-W)}>0 .
$$

(d) There exist numbers $r \geqslant 1, m \geqslant 1, \delta_{0}>0$ with the following property. 
For every $z \in \mathrm{C}$ and every number $\delta$ with $0<\delta<\delta_{0}$,

$$
\gamma(D(z, \delta)-W) \leqslant m \gamma(D(z, r \delta)-(W \cup E)) .
$$

(e) Given $f \in H^{\infty}(W)$ there is a sequence $\left\{F_{n}\right\}_{n=1}^{\infty}$ in $H^{\infty}(W)$ such that

(i) $F_{n}$ extends analytically to a neighborhood, depending on $n$, of $E$;

(ii) $\left|F_{n}\right| \leqslant\|f\|$ on $W$ and on this neighborhood;

(iii) $\left\{F_{n}\right\}$ converges to $f$ uniformly on each subset of $W$ at a positive distance from $E$.

Proof. We will show that $\mathrm{d} \Rightarrow \mathrm{e}$ and $\mathrm{d} \Rightarrow \mathrm{a} \Rightarrow \mathrm{b} \Rightarrow \mathrm{c} \Rightarrow \mathrm{d}$. If $m(E)=0$ we conclude with the implication $e \Rightarrow d$. We wish to begin with the demonstrations that $d \Rightarrow e$ and $d \Rightarrow$ a since they will be very similar, and we will use Vitushkin's approximation procedure.

Assume $d$ is true and let $f \in H^{\infty}(W)$. We extend $f$ to $\mathrm{C}^{*}$ by setting $f \equiv 0$ off $W$. Let $0<\delta<\delta_{0}$ and form Vitushkin's partition of unity $\left\{\phi_{k}\right\}_{k=1}^{\infty}$ corresponding to the number $\delta$. See $\S 2$ of [3] as a reference. (We will write $D_{k}$ for $\Delta_{k}$.) Let $\left\{D_{k}: k=1,2, \ldots, N(\delta)\right\}$ be an enumeration of all discs of Vitushkin's construction which contain points within $\delta$ of $E$. We have $\phi \equiv \phi_{1}+\cdots+\phi_{N(\delta)}$ $\equiv 1$ on the $\delta$-neighborhood of $E$. Let $f_{k}=T_{\phi_{k}} f$ where we again use $\S 2$ of [3] as a reference. Then $f_{k}$ is a bounded Borel measurable function on $\mathbf{C}^{*}$, which is analytic off a compact subset of $D_{k}-W$, and which is zero at $\infty$. We have the bound $\left\|f_{k}\right\| \leqslant 160\|f\|$. Since $\phi \equiv 1$ on the $\delta$-neighborhood of $E, f-\Sigma f_{k}$ is analytic there, which we may see by examining $T_{\phi} f$.

We wish to produce functions $g_{k}$ which approximate the $f_{k}$, and for this we split the indices $1,2, \ldots, N(\delta)$ into two classes, depending on whether or not $\gamma\left(r D_{k}-(W \cup E)\right)$ is zero. If this is zero, then (*) says that $\gamma\left(D_{k}-W\right)=0$. Since $f_{k}$ is bounded and analytic off a compact subset of $D_{k}-W, f_{k}$ extends analytically to $C^{*}$. (Compact sets of zero analytic capacity are removable singularities for bounded analytic functions.) Thus $f_{k} \equiv f_{k}(\infty)=0$, and in this case we take $g_{k}=0$.

On the other hand, suppose $\gamma\left(r D_{k}-(W \cup E)\right) \neq 0$. If one defines analytic diameters and analytic centers as in $\S$ VIII. 6 of [4], then it is possible to proceed as on pp. 101-102 of [20] to produce bounded measurable functions $g_{k}$, analytic off compact subsets of the $r D_{k}-(W \cup E)$, such that $\left\|g_{k}\right\| \leqslant M(m, r)\|f\|$, and such that $g_{k}-f_{k}$ has a triple zero at $\infty$. Here, $M(m, r)$ is a constant depending only on $m$ and $r$, and if we assume that $r \geqslant 2$ is an integer, we may take $M(m, r)=$ absolute constant $\cdot m r^{5}$. This assumption in no way harms the statement of the theorem. We write $M_{1}=M(m, r)$.

If $z_{k}$ is the center of $D_{k}$, we have $\left|z-z_{k}\right|^{3}\left|f_{k}(z)-g_{k}(z)\right| \leqslant$ $r^{3} \delta^{3}\left(160+M_{1}\right)\|f\|$ on the boundary of $r D_{k}$. This inequality is valid off $r D_{k}$ by the Maximum Modulus Principle, and is also true on $r D_{k}$. Thus it holds on 
C*. We will use this to estimate $\Sigma\left|f_{k}-g_{k}\right|$.

Fix $z \in \mathrm{C}$, and for each positive integer $j$, let $C_{j}$ be the circle with center $z$ and radius $j \delta$. Then each disc $D_{k}$ meets at least one and at most two of the $C_{j}$, except that if $z$ happens to be the center of one of the $D_{k}$, then that particular $D_{k}$ will not meet any of the $C_{j}$. Let $N_{j}$ be the number of discs $D_{k}$ which meet $C_{j}$. From the construction of the $D_{k}$, an arbitrary disc of radius $\delta$ can meet at most 64 of the $D_{k}$. Since $C_{j}$ can be covered by $10 j$ discs of radius $\delta$, each of which meets at most 64 of the $D_{k}$, we see that $N_{j} \leqslant 640 j$. Recalling that at most one disc $D_{k}$ fails to meet a circle $C_{j}$, we find

(1) $\sum_{k=1}^{N(\delta)}\left|f_{k}(z)-g_{k}(z)\right| \leqslant\left(160+M_{1}\right)\|f\|\left[1+N_{1}+\sum_{j=2}^{\infty} N_{j} /(j-1)^{3}\right] \leqslant M_{2}\|f\|$, since $\left|z-z_{k}\right| \geqslant r(j-1) \delta$ whenever $j \geqslant 2$ and $D_{k}$ meets $C_{j}$.

Now let us suppose that $\operatorname{dist}(z, E)>d>0$. Then if $\delta$ is chosen less than $d / 4 r$ we have $\operatorname{dist}\left(z, r D_{k}\right)>1 / 2 d$ for $k=1,2, \ldots, N(\delta)$. Then $j \leqslant d / 4 r \delta$ implies $N_{j}=0$, while $j>d / 4 r \delta>1$ implies $j \geqslant 2$, which in turn implies that $1 /(j-1)$ $\leqslant 2 / j$, and (1) becomes

$$
\sum_{k=1}^{N(\delta)}\left|f_{k}(z)-g_{k}(z)\right| \leqslant\left(160+M_{1}\right)\|f\| \sum_{j>d / 4 r \delta} 8 N_{j} / j^{3}
$$

$$
\begin{aligned}
& \leqslant\left(160+M_{1}\right)\|f\| \cdot 8(4 r \delta / d)^{3} \sum_{j>d / 4 r \delta} N_{j} \\
& \leqslant M_{3}\|f\|(\delta / d)^{3} \sum_{j=1}^{\infty} N_{j} \leqslant M_{3}\|f\|(\delta / d)^{3} \cdot 2 N(\delta)
\end{aligned}
$$

since each disc $D_{k}$ meets at most two of the $C_{j}$. We recall that $N(\delta)$ is the number of discs with which we originally covered $E$. Each of these discs contains points within $\delta$ of $E$, and so the union of these discs lies inside some disc $D$ whose radius is diam $E+6 \delta$. By the construction of the $D_{k}$, each point of $D$ is covered at most 25 times by $\bigcup D_{k}$. Therefore $N(\delta) \cdot \pi \delta^{2} \leqslant 25 \pi(\operatorname{diam} E+6 \delta)^{2}$, and inequality (2) becomes

$$
\sum_{k=1}^{N(\delta)}\left|f_{k}(z)-g_{k}(z)\right| \leqslant M_{4}\|f\| d^{-3} \cdot \delta(\operatorname{diam} E+6 \delta)^{2} .
$$

Now we define $F_{\delta}=f-\Sigma_{k=1}^{N(\delta)}\left(f_{k}-g_{k}\right)$. This function is analytic in $W$ and in a neighborhood of $E$ (because $f-\Sigma f_{k}$ is analytic in a neighborhood of $E$ ). It is bounded on $\mathrm{C}$, hence on $\mathrm{C}^{*}$, by $\left(1+M_{2}\right)\|f\|$, where $M_{2}=M_{2}(m, r)$. Finally $F_{\delta}$ $\rightarrow f$ uniformly in $z$, as $\delta \rightarrow 0$, provided $z$ remains at a positive distance (at least $d$ ) from $E$. This is almost the result we are after, and that result follows from this one and the Reduction of Norm Theorem. We remark that the compactness of $E$ and the 
the hypothesis $R(E)=C(E)$ are used only at this last step. This proves the implication $\mathrm{d} \Rightarrow \mathrm{e}$.

The implication $d \Rightarrow a$ is obtained in the same way, so we will only point out the differences. Again we assume that $d$ is true and that $r \geqslant 2$. Let $G$ be a bounded open set, let $\epsilon>0$, let $K \subset G-W$ be compact with $\gamma(K) \geqslant$ $\gamma(G-W)-\epsilon$, and let $f$ be an arbitrary element of $H^{\infty}\left(C^{*}-K\right)$. Since the compact set $K$ is contained in the open set $G$, there is a number $\eta$ with $0<\eta<$ $\operatorname{dist}(K, \mathrm{C}-G)$. Let $0<\delta<\min \left\{\delta_{0}, \eta / 4 r\right\}$. Now we proceed as above. Set $f \equiv 0$ on $K$. Form the partition of unity corresponding to $\delta$, and let $\left\{D_{k}: k=\right.$ $1,2, \ldots, N(\delta)\}$ be the discs which satisfy $\operatorname{dist}\left(D_{k}, E \cap K\right)<\delta$. Define $f_{k}$ as before. Since $\Sigma \phi_{k} \equiv 1$ on the $\delta$-neighborhood of $E \cap K, f-\Sigma f_{k}$ is analytic there, and since each $f_{k}$ is analytic on the neighborhood $\mathrm{C}^{*}-K$ of $E-K$, $f-\Sigma f_{k}$ is actually analytic on a neighborhood of $E$.

Define $g_{k}$ as before. Each function $g_{k}$ is analytic off a compact subset of $r D_{k}-(W \cup E)$, but that is not enough. We need to know that $g_{k}$ is analytic off a compact subset of $G$, and we show now that this is true. Let $z_{k}$ be the center of $D_{k}$. Since $D_{k}$ contains points within $\delta$ of $E \cap K$, let $w_{k} \in D_{k}$ and $e_{k} \in E \cap K$ satisfy $\left|w_{k}-e_{k}\right|<\delta$. Then for each point $z$ belonging to $r D_{k}$ we have $\left|z-e_{k}\right| \leqslant\left|z-z_{k}\right|+\left|z_{k}-w_{k}\right|+\left|w_{k}-e_{k}\right| \leqslant r \delta+\delta+\delta=(r+2) \delta \leqslant$ $2 r \delta \leqslant 1 / 2 \eta$. So every point of every disc $r D_{k}$ lies within $1 / 2 \eta$ of $E \cap K$, and therefore lies inside $G$. If we write $N$ for the $1 / 2 \eta$-neighborhood of $K$, then $g_{k}$ is analytic off a compact subset of $\bar{N}-(W \cup E) \subset G-(W \cup E)$.

Therefore the function $F_{\delta}$ is analytic on $W$, on a neighborhood of $E$, and off the compact subset $\bar{N}$ of $G$. This function is bounded on $\mathrm{C}^{*}$ by $\left(1+M_{2}\right)\|f\|$, and if we take a sequence $\delta_{n} \downarrow 0$, then the corresponding functions $F_{n}=F_{\delta_{n}}$ converge uniformly to $f$ on any set at positive distance from $E \cap K$. Let $U$ be the open set $W \cup\left(C^{*}-\bar{N}\right)$ and let $Q$ be the compact set $E \cap \bar{N}$. We have $Q \subset$ C - $U$, and since $R(E)=C(E)$ we also have $R(Q)=C(Q)$ by Tietze's Extension Theorem. Let $J=H^{\infty}\left(\mathbf{C}^{*}-K\right)$, a closed subalgebra of $H^{\infty}(U)$. The Reduction of Norm Theorem tells us that for each $f \in J$ we can find a sequence $\left\{F_{n}\right\}$ in $H^{\infty}(U)$ with $F_{n}$ analytic on a neighborhood of $Q$, such that $\left\|F_{n}\right\| \leqslant\|f\|$, and $F_{n} \rightarrow f$ uniformly on any subset of $U$ at positive distance from $Q$.

Let $h$ be the Ahlfors function for $K$. Then the restriction of $h$ to $\mathrm{C}^{*}-K$ belongs to $J$, and we have a sequence $\left\{H_{n}\right\}$ of functions "admissible" for compact subsets of $\bar{N}-[W \cup(E \cap \bar{N})]=\bar{N}-[W \cup E] \subset G-[W \cup E]$. Choose $R>0$ so large that $E \cup G \subset D(0,1 / 2 R)$ and that the circle $C$ with center 0 and radius $R$ lies in $W$. Choose a positive integer $n$ so that $\left\|H_{n}-h\right\|_{C} \leqslant \epsilon / R$. This yields $\gamma(G-W) \leqslant \gamma(K)+\epsilon=h^{\prime}(\infty)+\epsilon \leqslant\left|H_{n}^{\prime}(\infty)\right|+2 \epsilon \leqslant \gamma(G-(W \cup E))+2 \epsilon$. Since $\epsilon$ is arbitrary, the proof of $d \Rightarrow a$ is complete.

The implications $a \Rightarrow b \Rightarrow c$ are trivial. To obtain $c$ from $b$, take $r(z)=1$ 
for every $z$. The proof of (the contrapositive of) the implication $c \Rightarrow d$ is so very similar to the corresponding portion of Vitushkin's Theorem, that we will omit it, except for one remark. The proof proceeds by repeated application of (the contrapositive of) the implication $\mathrm{d} \Rightarrow \mathrm{a}$, which we just established. This implication is applied to various open sets, and we must make sure that each of these open sets is a neighborhood of $\infty$. We choose $R \geqslant 10$ so that $E \subset D(0, R)$ and $C^{*}-D(0, R) \subset W$. Then we add on $C^{*}-\bar{D}(0,2 R)$ to each of the open sets. This is a minor technical point which does no damage because everything takes place well inside $D(0, R)$.

The only implication remaining is that $\mathrm{e} \Rightarrow \mathrm{d}$ whenever $m(E)=0$. This will be an imitation of Lemma 8.2 of [6]. Let $r>1$ and $\delta_{0}>0$ be arbitrary and let $m=240 r /(r-1)$. Let $D$ be any open disc of radius $\delta$. Let $f$ be a function admissible for a compact subset of $D-W$. Since $f$ is analytic on $W$, there is a sequence $\left\{f_{n}\right\}$ associated to $f$ by the hypothesis e. Let $W_{n}$ be a neighborhood of $E$ on which $f_{n}$ is analytic. Choose $R>0$ so that $r D \subset D(0, R)$ and $C^{*}-D(0, R) \subset W$. Let $\epsilon>0$ and let $G$ be a neighborhood of $E$ such that $m(G) \leqslant \pi \epsilon^{2} \delta^{2}(r-1)^{2} / 6400\left(4 R^{2}\right)$. Redefine $f_{n}$ so that $f_{n} \equiv 0$ off $W \cup\left(G \cap W_{n}\right)$. Since the sequence $\left\{f_{n}\right\}$ lies in the closed unit ball of $L^{\infty}(d m)$, some subsequence, which we will call $\left\{f_{n}\right\}$, converges weak star in $L^{\infty}(d m)$ to a limit function in the closed unit ball. We write this limit function as $f+h$, where $h \in L^{\infty}(d m)$. Then $\|h\|_{\infty} \leqslant 2$ and $h=0$ a.e. on $W$.

Define $\tilde{h} \in L^{\infty}(d m)$ by $\tilde{h} \equiv 0$ on $W \cup G$ and $\tilde{h} \equiv h$ off $W \cup G$. Let $\phi$ be a continuously differentiable function on $C^{*}$, with $0 \leqslant \phi \leqslant 1, \phi \equiv 1$ on $\bar{D}$, $\phi \equiv 0$ off $r D$, and $\|\operatorname{grad} \phi\| \leqslant 20 /(r-1) \delta$. Consider the functions $T_{\phi} f_{n}$ and the function $\widetilde{H}=T_{\phi} \widetilde{h}-\widetilde{h} \phi$. The functions $T_{\phi} f_{n}$ are bounded Borel functions which are analytic off a compact subset of $r D$, analytic on $W \cup W_{n}$, and zero at $\infty$. Each is bounded in modulus by $160 r /(r-1)$. The function $\widetilde{H}$ is the convolution of a bounded measurable function and a locally integrable function, and is continuous on $\mathbf{C}^{*}$ by the proof of Lemma 3.1.5 of [1]. From Theorem 10.7 of [14] we see that $\widetilde{H}$ is analytic on $\mathbf{C}-[\operatorname{supp} \phi-(W \cup G)]$. By Lemma 3.1 .1 of [1], $\widetilde{H}$ is bounded by $40 / \pi(r-1) \delta \cdot 2 \sqrt{\pi m(\operatorname{supp} \phi)}<80 r /(r-1)$, and so $\widetilde{H}$ is analytic on $\mathbf{C}^{*}-[\operatorname{supp} \phi-(W \cup G)]=W \cup G \cup\left(\mathbf{C}^{*}-\operatorname{supp} \phi\right)$. From the above bounds and the definition of capacity,

$$
\begin{aligned}
\left|\left(T_{\phi} f_{n}\right)^{\prime}(\infty)-\widetilde{H}^{\prime}(\infty)\right| & \leqslant \frac{240 r}{r-1} \gamma\left(\operatorname{supp} \phi-\left(W \cup\left(G \cap W_{n}\right)\right)\right) \\
& \leqslant \frac{240 r}{r-1} \gamma(r D-(W \cup E)) .
\end{aligned}
$$

Recall the choice of $R$. Let $C$ be the circle with center 0 and radius $2 R$. If $z \in C$ then $\operatorname{dist}(z, \operatorname{supp} \phi)>R$ and therefore the function of $w,(w-z)^{-1}$. (grad $\phi)$, belongs to $L^{\infty}(d m)$. If we split up the defining integrals as an integral 
over supp $\phi-G$ and an integral over supp $\phi \cap G$, we see that

$$
\begin{aligned}
T_{\phi} f_{n}(z)-\widetilde{H}(z) \rightarrow & \frac{1}{\pi} \iint \frac{f(w)}{w-z} \operatorname{grad} \phi d m(w) \\
& +\frac{1}{\pi} \int_{\operatorname{supp} \phi \cap G} \frac{h(w)}{w-z} \operatorname{grad} \phi d m(w) .
\end{aligned}
$$

The first integral here is immediately recognizable as $T_{\phi} f(z)-f(z) \phi(z)=T_{\phi} f(z)$ since $\phi(z)=0$. This is actually equal to $f(z)$ as we now show. $T_{\phi} f-f$ is analy tic on $D$ because $\phi \equiv 1$ there. Since $f$ is analytic off a compact subset of $D$, so is $T_{\phi} f$. Thus $T_{\phi} f-f$ is entire, hence constant by Liouville's Theorem. This constant is zero since that is the value of the function at $\infty$. Therefore, if $z \in C$,

$$
T_{\phi} f_{n}(z)-\widetilde{H}(z)-f(z) \rightarrow \frac{1}{\pi} \iint_{G} \frac{h(w)}{w-z} \operatorname{grad} \phi d m(w)
$$

since the integral over $G$ is the same as over $G \cap \operatorname{supp} \phi$.

For each $z \in C$ there is a positive integer $N(z)$ with the property that $\left|T_{\phi} f_{n}(z)-\widetilde{H}(z)-f(z)\right| \leqslant \epsilon / 2 R$ whenever $n \geqslant N(z)$. This follows from the choice of $G$ and from Lemma 3.1.1 of [1]. Since $C$ is compact and since $T_{\phi} f_{n}-\widetilde{H}-f$ is continuous on $C$, there is a positive integer $N$ so that this inequality holds for all $z \in C$ and all $n \geqslant N$. Since $T_{\phi} f_{n}-\widetilde{H}-f$ is analytic off a compact subset of $r D$,

$$
\left(T_{\phi} f_{n}\right)^{\prime}(\infty)-\widetilde{H}^{\prime}(\infty)-f^{\prime}(\infty)=(2 \pi i)^{-1} \int_{C}\left[T_{\phi} f_{n}(z)-\widetilde{H}(z)-f(z)\right] d z .
$$

If $n$ is large, the absolute value of this quantity is at most $\epsilon$, and then (4) implies that $\left|f^{\prime}(\infty)\right| \leqslant 240 r /(r-1) \cdot \gamma(r D-(W \cup E))+\epsilon$. Letting $\epsilon$ go to zero, and then letting $f$ vary, we find that $\gamma(D-W) \leqslant 240 r /(r-1) \cdot \gamma(r D-(W \cup E))$, as desired. This completes the proof that $\mathrm{e} \Rightarrow \mathrm{d}$ whenever $m(E)=0$, and completes the proof of Theorem 1 .

Corollary 1. Let $W \subset \mathrm{C}^{*}$ be an open set containing the point $\infty$, and let $E \subset \mathrm{C}-W$ be compact. Suppose that for each $z \in E$ we have

$$
\liminf _{\delta \rightarrow 0} \frac{\gamma(D(z, \delta)-(W \cup E))}{\delta}>0 .
$$

Then given $f \in H^{\infty}(W)$ there is a sequence $\left\{F_{n}\right\}_{n=1}^{\infty}$ in $H^{\infty}(W)$ such that

(i) $F_{n}$ extends analytically to a neighborhood, depending on $n$, of $E$;

(ii) $\left|F_{n}\right| \leqslant\|f\|$ on $W$ and on this neighborhood;

(iii) $\left\{F_{n}\right\}$ converges to $f$ uniformly on each subset of $W$ at a positive distance from $E$.

Proof. Using Theorem VIII.5.1 of [4], we see that $R(E)=C(E)$. Hypoth- 
esis $c$ of Theorem 1 is satisfied with $r=1$, and so Corollary 1 follows from the implication $\mathrm{c} \Rightarrow \mathrm{e}$ of Theorem 1 .

It will usually be in the form of this corollary that we make use of Theorem 1. We will give an example later of an instance in which Corollary 1 fails to give any information, while Theorem 1 does tell us what we want to know.

Vitushkin also has methods which allow one to approximate a single function rather than a whole Banach algebra of functions. Most of these methods make the transition from uniform approximation to pointwise bounded approximation. Our main difficulty in using these results is that we are no longer able to reduce norms. This means, for one thing, that we can no longer expect a lim inf condition, as the proof of $c \Rightarrow d$ in Theorem 1 made repeated use of the implication $d \Rightarrow a$ and the fact that the constant in part a is one. Theorem 2 is the analogue of Theorem IV.2.1 and Lemma IV.2.1 of [19], and Theorem 3 is the analogue of Theorem IV.2.2.

TheOREM 2. Let $W \subset \mathrm{C}^{*}$ be an open set containing the point $\infty$ and let $f \in H^{\infty}(W)$. Let $E$ be a compact set of measure zero which satisfies $\partial W \subset E \subset$ $(\mathbf{C}-\bar{W})-\subset \mathbf{C}-W$. (This requires that $\partial W$ have measure zero, and also says that every neighborhood of every point of $E$ meets $\mathbf{C}-\bar{W}$.) Then we have the implications $\mathrm{a} \Rightarrow \mathrm{b} \Leftrightarrow \mathrm{c}$, where:

(a) $\gamma(D-W) \leqslant m_{f} \gamma(D-(W \cup E))$ for every open disc $D$, with the constant $m_{f}$ independent of the choice of $D$.

(b) There exist constants $m \geqslant 1, r \geqslant 1, \delta_{0}>0$ with the following property. For every open disc $D$ of radius $\delta<\delta_{0}$ and for every continuously differentiable function $\phi$ with compact support contained in $D$, we have

$\left|\iint f(z) \operatorname{grad} \phi d m(z)\right| \leqslant m\|f\| \cdot \delta\|\operatorname{grad} \phi\| \cdot \gamma(r D-(W \cup E))$.

(c) There exists a sequence $\left\{F_{n}\right\}_{n=1}^{\infty}$ in $H^{\infty}(W)$ such that

(i) $F_{n}$ extends analytically to a neighborhood, depending on $n$, of $W \cup E$;

(ii) $\left|F_{n}\right| \leqslant M\|f\|$ there, with the constant $M$ independent of $n$;

(iii) $\left\{F_{n}\right\}$ converges to $f$ uniformly on each subset of $W$ at a positive distance from $E$.

Proof. We will prove that $b \Rightarrow c \Rightarrow b$ and that $a \Rightarrow b$. To show that $b \Rightarrow$ $c$ we make the same changes in Vitushkin's approximation procedure as we did in showing that $d \Rightarrow e$ in Theorem 1 . There is only one difference that enters here, and this occurs when we split up the indices and look at those $k$ for which $\gamma\left(r D_{k}-(W \cup E)\right)=0$. In the present situation, $r D_{k}-(W \cup E)=r D_{k}-(\bar{W} \cup E)$ is an open set of zero analytic capacity. It must therefore be empty, and $r D_{k} \subset$ $W \cup E$. We claim that, in fact, $r D_{k} \subset W$. For suppose there is a point $z \in r D_{k}$ $\cap E$. Since $r D_{k}$ is a neighborhood of $z \in E, r D_{k}$ meets the open set $C-\bar{W}$. 
Then there is a disc $\widetilde{D}$ contained in the intersection of these two open sets, and

$$
\begin{aligned}
\widetilde{D}-E & =\tilde{D} \cap(\mathrm{C}-E) \subset r D_{k} \cap(\mathrm{C}-\bar{W}) \cap(\mathrm{C}-E) \\
& =r D_{k} \cap[\mathrm{C}-(\bar{W} \cup E)]=r D_{k} \cap[\mathrm{C}-(W \cup E)] \\
& =r D_{k}-(W \cup E)=\text { the empty set. }
\end{aligned}
$$

This contradicts the hypothesis that $E$ has measure zero. Therefore $r D_{k}$ does not meet $E, r D_{k} \subset W$, and $D_{k} \subset W$. Since $D_{k}-W$ is empty, and $f_{k}$ is bounded and analytic off a compact subset of $D_{k}-W$, we must have $f_{k} \equiv f_{k}(\infty)=0$. So we take $g_{k} \equiv 0$.

Next we show that $\mathrm{c} \Rightarrow \mathrm{b}$. Let $r=1, \delta_{0}=1, m=8 \pi M$, where $M$ is the constant of part c. Choose $D$ and $\phi$. If $\|\operatorname{grad} \phi\|$ is 0 or $\infty$ there is nothing to prove, so we assume it is equal to $q$, with $0<q<\infty$. Let $\epsilon>0$. Choose $\eta>0$ so that $N$, the $\eta$-neighborhood of $E$, has measure $m(N) \leqslant \epsilon / 2(M+1) q\|f\|$.

Next choose $F$ to be one of the $F_{n}$ so that $F$ is analytic on $G \supset W \cup E,|F| \leqslant$ $M\|f\|$ on $G$, and $|F-f| \leqslant \epsilon / 2 \pi q \delta^{2}$ on $W-N$. Define $H \equiv F$ on $W \cup(G \cap N)$ and $H \equiv 0$ elsewhere. Also define $f \equiv 0$ off $W$. Consider the function $T_{\phi} H$. This is analytic off a compact subset of $D-(W \cup E)$ and is bounded by $8 \delta q M\|f\|$. We have

$$
\begin{aligned}
\left(T_{\phi} H\right)^{\prime}(\infty) & =\frac{1}{2 \pi i} \int_{\partial D} \frac{1}{\pi} \iint \frac{H(w)}{w-z} \operatorname{grad} \phi d m(w) d z \\
& =\frac{1}{\pi} \iint_{D} H(w) \operatorname{grad} \phi d m(w) .
\end{aligned}
$$

Now,

$$
\begin{aligned}
\iint_{D}[f(w)-H(w)] \operatorname{grad} \phi d m(w) & =\iint_{D \cap[W \cup(G \cap N)]} \\
& =\iint_{(D \cap[W \cup(G \cap N)])-N}+\iint_{D \cap[W \cup(G \cap N)] \cap N}
\end{aligned}
$$

From the three inclusions:

$$
\begin{gathered}
(D \cap[W \cup(G \cap N)])-N \subset W-N, \\
D \cap[W \cup(G \cap N)] \subset W \cup(G \cap N)=[W \cup(G \cap N)] \cap G, \\
D \cap[W \cup(G \cap N)] \cap N \subset N,
\end{gathered}
$$

we see that the last two integrals above are bounded in absolute value by $\epsilon / 2 \pi q \delta^{2} \cdot q \pi \delta^{2}=\epsilon / 2$ and $(M+1)\|f\| \cdot q \cdot \epsilon / 2(M+1) q\|f\|=\epsilon / 2$ respectively. Therefore 


$$
\begin{aligned}
\mid \iint f(w) & \operatorname{grad} \phi d m(w)|=| \iint_{D} f(w) \operatorname{grad} \phi d m(w) \mid \\
& \leqslant\left|\iint_{D} H(w) \operatorname{grad} \phi d m(w)\right|+\left|\iint_{D}[f(w)-H(w)] \operatorname{grad} \phi d m(w)\right| \\
& \leqslant \pi\left|\left(T_{\phi} H\right)^{\prime}(\infty)\right|+\epsilon \leqslant 8 \pi \delta q M\|f\| \gamma(D-(W \cup E))+\epsilon \\
& =m\|f\| \cdot \delta\|\operatorname{grad} \phi\| \cdot \gamma(D-(W \cup E))+\epsilon .
\end{aligned}
$$

Since $\epsilon$ is arbitrary we are finished. Thus $b$ and $c$ are equivalent.

Now suppose that a is true. Let $r=1, \delta_{0}=1, m=8 m_{f}$. Choose $D$ and $\phi$. Extend $f$ to $\mathbf{C}^{*}$ by setting it equal to zero off $W$. Then $T_{\phi} f$ is defined, is analytic off a compact subset of $D-W$, and is bounded by $8 \delta\|\operatorname{grad} \phi\|\|f\|$. Thus $\left|\left(T_{\phi} f\right)^{\prime}(\infty)\right| \leqslant 8 \delta\|\operatorname{grad} \phi\|\|f\| \gamma(D-W) \leqslant 8 m_{f}\|f\| \cdot \delta\|\operatorname{grad} \phi\| \cdot$ $\gamma(D-(W \cup E))$. Since $\left(T_{\phi} f\right)^{\prime}(\infty)$ is the integral in $b, b$ is true. This completes the proof of Theorem 2 .

We remark that it is possible to replace the hypothesis that $E$ have measure zero by the less restrictive requirement that $E$ have no interior. This allows us to include situations in which $\partial W$ may have positive area. In this case the conclusion of Theorem 2 is that $a \Rightarrow b \Rightarrow c$, and the proof proceeds as above.

In Vitushkin's work the extra condition on $E$ does not explicitly appear, but it is there. If $K$ is a compact set, Vitushkin considers $E=\partial K$, and the condition reduces to the tautology $\partial($ interior of $K) \subset \partial K \subset\left(\mathbf{C}-(\text { interior of } K)^{-}\right)^{-}$.

Finally we note that we cannot show equivalence between part a and either $\mathrm{b}$ or $\mathrm{c}$; knowing about the one function $f$ is just not enough.

THEOREM 3. Let $f$ be continuous on $\mathbf{C}^{*}$ with modulus of continuity $\omega(\delta)$, and let $E \subset \mathrm{C}^{*}$ be any set. Assume that there exist numbers $\tilde{m} \geqslant 1$, $\tilde{\delta}_{0}>0$ with the property that $\left|\int_{\partial Q} f(z) d z\right| \leqslant \tilde{m} \omega(\delta) \gamma(Q-E)$ for all squares $Q$ of side $\delta<\tilde{\delta}_{0}$. Then there exist constants $m \geqslant 1, r \geqslant 1, \delta_{0}>0$ with the following property. For every open disc $D$ of radius $\delta<\delta_{0}$ and every continuously differentiable function $\phi$ with compact support contained in $D$, we have

$$
\begin{aligned}
\left|\iint f(z) \operatorname{grad} \phi d m(z)\right| & \leqslant m \omega(2 \delta) \cdot \delta\|\operatorname{grad} \phi\| \cdot \gamma(r D-E) \\
& \leqslant 2 m\|f\| \cdot \delta\|\operatorname{grad} \phi\| \cdot \gamma(r D-E) .
\end{aligned}
$$

This is proved exactly as Theorem IV.2.2 of [19] , and continuity is essential. Vitushkin, who works with uniform approximation, is able to obtain a converse. From a statement about pointwise bounded approximation, which is what we have here according to Theorem 2, we have not been able to produce a converse. 
2. When does $\gamma(V)=\gamma(\bar{V})$ ? There are examples of bounded open sets $V$ with $\gamma(V)<\gamma(\bar{V})$. However we can use Corollary 1 to obtain equality in many cases.

THEOREM 4. Let $V$ be a bounded open set. If at least one of the following conditions holds, then $\gamma(V)=\gamma(\bar{V})=\alpha(V)=\alpha(\bar{V})$.

(a) $V$ has finitely many components.

(b) The diameters of the components of $V$ are bounded away from zero.

(c) $\partial V$ coincides with the union of the boundaries of the components of $V$.

(d) Every point of $\partial V$ is either a boundary point of a component of $V$, or is a cluster point of a sequence of components of $V$ whose diameters are bounded away from zero.

Moreover, conditions $\mathrm{c}$ and $\mathrm{d}$ can be weakened to allow part of $\partial \mathrm{V}$ to be what Gamelin and Garnett $[6, \S 7]$ call a o-curvilinear null set, that is, a countable union of bounded sets $B_{j}$, each of which lies on a $C^{2}$ curve $C_{j}$, and has zero outer arc length. (Any countable set is a $\sigma$-curvilinear null set, for example.)

Proof. Let $W=C^{*}-\bar{V}$ and let $E=\partial V$. Let $z \in E$. If $z$ is a boundary point of one or more components of $V$, choose one such component and let $\delta_{0}$ be the diameter of that component. If $z$ does not belong to the boundary of any component of $V$, choose a sequence of components which cluster at $z$, and whose diameters are bounded away from zero, and let $\delta_{0}$ be the infimum of those diameters. If $0<\delta<\delta_{0}$ then $D(z, \delta) \cap V$ contains an $\operatorname{arc} A$ of diameter $1 / 2 \delta$. Since the analytic capacity of a continuum is at least one fourth the diameter, $\gamma(D(z, \delta)-(W \cup E)) / \delta=\gamma(D(z, \delta) \cap V) / \delta \geqslant \gamma(A) / \delta \geqslant 1 / 8$. Thus Corollary 1 applies.

Let $V \subset D(0, R)$ and let $C$ be the circle with center 0 and radius $2 R$. Let $f$ be the Ahlfors function for $\bar{V}$ and let $\epsilon>0$. Let $F$ be one of the functions $F_{n}$ of Corollary 1 so that $\|F-f\|_{C} \leqslant \epsilon / 2 R$. Then $F$ is "admissible" for a compact subset of $V$, and $\gamma(\bar{V})=f^{\prime}(\infty) \leqslant\left|F^{\prime}(\infty)\right|+\epsilon \leqslant \gamma(V)+\epsilon$. Since $\epsilon$ is arbitrary, $\gamma(\bar{V}) \leqslant \gamma(V)$, and therefore $\gamma(\bar{V}) \leqslant \gamma(V)=\alpha(V) \leqslant \alpha(\bar{V}) \leqslant \gamma(\bar{V})$.

For the case of finitely many components we mention two other proofs. The first appears in [15] and uses completely different methods. The second was communicated to the author by T. Gamelin. The condition of Theorem 7(vi) of [5], with $K=\mathrm{C}^{*}-V, E=$ the empty set, is satisfied and so $R(K)$ is a hypodirichlet algebra on $\partial K=\partial V$. From here one can proceed as before, approximating the Ahlfors function for $\bar{V}$, using part (iv) of that theorem. Gamelin has also pointed out that in cases $a$ and $b$ it is possible to use Corollary VIII.10.6 of [4] to produce a sequence of approximating functions. Finally, all four conditions can be taken care of by Theorem 8.9 and the last paragraph on p. 389 of [6], and here is where the remark about $\sigma$-curvilinear null sets enters. 
Since we plan to concentrate next on the types of boundary points described in this theorem, we define, for any bounded open set $V, S=S(V)=$ the union of the boundaries of the components of $V$, and $S_{0}=S_{0}(V)=$ the union of $S(V)$ and all boundary points of $V$ which are cluster points of sequences of components of $V$ whose diameters are bounded away from zero. For convenience we also introduce the notation $N(A, \delta)$ for the $\delta$-neighborhood of the set $A \subset$ C.

We have just seen that the situation in which the boundary of $V$ was equal to $S$ or $S_{0}$ was a very nice one. We were able to add $\partial V$ on to $V$, or, equivalently, remove $\partial V$ from $\bar{V}$, without affecting the numerical value of the analytic capacity. Of course it follows that part of $\partial V$ can also be added on to $V$ or subtracted from $\bar{V}$ with the same result. Now we consider the situation in which $\partial V$ is not equal to $S_{0}$. Again, there are examples of open sets $V$ with $\gamma(V)<$ $\gamma(\bar{V})$, so it is too much to hope for the possibility that all of $\partial V$ can be added or removed. But what about part of $\partial V$ ? Specifically, what about the set $S_{0}$ ? On $S_{0}$ the lim inf condition is satisfied, so we would expect that $S_{0}$ can be added or removed. However $S_{0}$ is not a compact set because $S_{0} \neq \partial V=\bar{S}_{0}$, and so the results above do not apply to $S_{0}$. They do apply to compact subsets $K$ of $S_{0}$, and so we have the result that $\gamma(\bar{V})=\gamma(\bar{V}-K)$.

Is it also true that $\gamma(V)=\gamma(V \cup K)$ ? This is not immediately obvious. The trouble here is that $V \cup K$ is not compact, and so to look at $\gamma(V \cup K)$ we must look at $\gamma(L)$ where $L$ is a compact subset of $V \cup K$. The set $L$ may not contain enough of $V$ to produce the arcs which previously gave us our capacity estimate.

What we plan to do in this section is to show that $\gamma(V)=\gamma\left(V \cup S_{0}\right)$ and $\gamma(\bar{V})=\gamma\left(\bar{V}-S_{0}\right)$ for all bounded open sets $V$. This will include the above statements about $K \subset S_{0}$. The following lemma and Theorem 5 were given by the referee, Donald Wilken, and improve the author's original Theorem 5, which had been stated and proved only for a certain class of open sets. These are the sets of type I, which will be defined later.

LEMMA. Let $V$ be a bounded open set and let $L \subset V \cup S_{0}(V)$ be compact. Given $\delta>0$, there exists a bounded open set $U$ with

(a) $U \subset V \cap N(L, \delta)$;

(b) $L \subset \bar{U}$, which says that $U$ is not empty if $L$ is not empty;

(c) $\partial U=S_{0}(U)$, which says $\gamma(U)=\gamma(\bar{U})=\alpha(U)=\alpha(\bar{U})$ via Theorem 4d.

Proof. Let $O_{n}$ be the union of all components of $V$ with diameters at least $\delta / n$. Then $V \cup S_{0}(V)=\cup \bar{O}_{n}$. Let $L_{n}=L \cap \bar{O}_{n}$ and $G_{n}=V \cap$ $N\left(L_{n}, \delta / n\right)$. Let $\left\{U_{n, j}\right\}$ be the collection of all components of $U_{n}$ with diameters at least $\delta / 2 n$, and let $U_{n}$ be the union of this collection. Let $U$ be the 
union of the $U_{n}$. Part a follows immediately; part b follows from the relation $L_{n} \subset \bar{U}_{n}$; and part c follows from the relation $\partial U_{n}=S_{0}\left(U_{n}\right)$.

THEOREM 5. If $V$ is any bounded open set, then $\gamma(V)=\gamma\left(V \cup S_{0}\right)=$ $\alpha(V)=\alpha\left(V \cup S_{0}\right)$ and $\gamma(\bar{V})=\gamma\left(\bar{V}-S_{0}\right)$.

Proof. The first string of equalities follows immediately from the lemma. Given $L \subset V \cup S_{0}$ compact, we take $\delta=1$ and the lemma yields a set $U$ with $\gamma(L) \leqslant \gamma(\bar{U})=\gamma(U) \leqslant \gamma(V)$. Taking the supremum over $L$ we see that $\gamma\left(V \cup S_{0}\right) \leqslant \gamma(V)=\alpha(V) \leqslant \alpha\left(V \cup S_{0}\right) \leqslant \gamma\left(V \cup S_{0}\right)$. The second part of the theorem requires some work.

Let $\epsilon>0$, let $V \subset D(0, R)$, and let $C$ be the circle with center at the origin and radius $2 R$. For each positive integer $n$, let $O_{n}$ be the union of all those components of $V$ with diameters at least $1 / n$. Then $O_{1} \subset O_{2} \subset O_{3} \subset \cdots$.

We apply Corollary 1 with $W_{1}=\mathrm{C}^{*}-\bar{V}, E_{1}=\partial O_{1}$, and $f=g_{1}=$ Ahlfors function for $\bar{V}$. This yields a function $g_{2}$, which is one of the $\left\{F_{n}\right\}$, such that $g_{2}$ is analytic in a neighborhood $G_{2} \supset W_{1} \cup \partial O_{1}$, with $\left|g_{2}\right| \leqslant 1$ on $G_{2}$ and $\left\|g_{2}-g_{1}\right\|_{C} \leqslant \epsilon / 4 R \cdot 1 / 2$. We apply the lemma with $V, L_{2}=\partial O_{2}-G_{2}$, and $\delta_{2}=1 / 4 \operatorname{dist}\left(\partial O_{1}, \partial G_{2}\right)$. This yields a set $U_{2} \subset V \cap N\left(L_{2}, \delta_{2}\right)$ with $L_{2} \subset \bar{U}_{2}$ and $\partial U_{2}=S_{0}\left(U_{2}\right)$.

We set $W_{2}=G_{2}-\bar{U}_{2}, E_{2}=\partial U_{2}-W_{2}$ and we observe that:

(i) $W_{2} \cup E_{2}=\left(G_{2}-\bar{U}_{2}\right) \cup \partial U_{2}=\left(G_{2}-U_{2}\right) \cup \partial U_{2} \subset \mathrm{C}^{*}-U_{2}$;

(ii) $W_{1}=W_{1}-\bar{V} \subset G_{2}-\bar{V} \subset G_{2}-\bar{U}_{2}=W_{2}$;

(iii) $\partial O_{1} \cup \partial O_{2}=\partial O_{2} \subset W_{2} \cup E_{2}$.

Corollary 1 applies to $W_{2}, E_{2}, g_{2}$ because every $z \in E_{2} \subset S_{0}\left(U_{2}\right)$ satisfies $\gamma\left(D(z, \delta)-\left(W_{2} \cup E_{2}\right)\right) \geqslant \gamma\left(D(z, \delta) \cap U_{2}\right) \geqslant \delta / 8$ for small positive $\delta<\delta(z)$.

When we apply Corollary 1 , we obtain a function $g_{3}$ analytic on an open set $G_{3} \supset W_{2} \cup E_{2} \supset W_{1} \cup \partial O_{1} \cup \partial O_{2}$ with $\left|g_{3}\right| \leqslant 1$ on $G_{3}$ and $\left\|g_{3}-g_{2}\right\|_{C} \leqslant$ $\epsilon / 4 R \cdot 1 / 4$. Next we apply the lemma with $V, L_{3}=\partial O_{3}-G_{3}$, and $\delta_{3}=$ $\min \left\{1 / 2 \delta_{2}, 1 / 4 \operatorname{dist}\left(\partial O_{1} \cup \partial O_{2}, \partial G_{3}\right)\right\}$, and this yields a set $U_{3} \subset V \cap N\left(L_{3}, \delta_{3}\right)$ with $L_{3} \subset \bar{U}_{3}$ and $\partial U_{3}=S_{0}\left(U_{3}\right)$.

We then set $W_{3}=G_{3}-\bar{U}_{3}$ and $E_{3}=\partial U_{3}-W_{3}$ and proceed as above. We find (i) $W_{3} \cup E_{3} \subset \mathrm{C}^{*}-U_{3}$; (ii) $W_{1} \subset W_{3}$; (iii) $\partial O_{1} \cup \partial O_{2} \cup \partial O_{3}=$ $\partial O_{3} \subset W_{3} \cup E_{3}$.

Continuing in this manner, we define $d_{n}=1 / 2 \operatorname{dist}\left(\partial O_{n}, \partial G_{n+1}\right)=$ $1 / 2 \operatorname{dist}\left(\partial O_{1} \cup \cdots \cup \partial O_{n}, \partial G_{n+1}\right)$ and

$$
\delta_{n}=\min \left\{1 / 2 \delta_{n-1}, 1 / 4 \operatorname{dist}\left(\partial O_{1} \cup \cdots \cup \partial O_{n-1}, \partial G_{n}\right)\right\},
$$

and this produces a sequence $\left\{g_{n}\right\}_{n=1}^{\infty}$ of functions and a sequence $\left\{W_{n}\right\}_{n=1}^{\infty}$ of open sets which satisfy the following conditions:

(a) $\left(\mathbf{C}^{*}-\bar{V}\right) \cup \partial O_{1} \cup \cdots \cup \partial O_{n}=W_{1} \cup \partial O_{n} \subset W_{n+1}$; 
(b) $g_{n}$ is analytic and bounded by 1 in $W_{n}$;

(c) $\left\|g_{n}-g_{n-1}\right\|_{C} \leqslant \epsilon / 4 R \cdot 2^{-n}$;

(d) $N\left(\partial O_{n}, d_{n}\right)$ is contained in each of the sets $W_{n+1}, W_{n+2}, \ldots$.

Only the last of these assertions requires any elaboration, and it is verified without much difficulty by the choice of $d_{n}$ and $\delta_{n}$, and by the construction of the sets $W_{n}$ as:

$$
\begin{aligned}
& W_{1} \cup \partial O_{1} \cup \partial O_{2} \cup \cdots \cup \partial O_{n} \subset W_{n} \cup E_{n} \subset G_{n+1} ; \\
& W_{n+1}=G_{n+1}-\bar{U}_{n+1} ; \\
& W_{1} \cup \partial O_{1} \cup \partial O_{2} \cup \cdots \cup \partial O_{n+1} \subset W_{n+1} \cup E_{n+1} \subset G_{n+2} \\
& W_{n+2}=G_{n+2}-\bar{U}_{n+2} .
\end{aligned}
$$

Now we set $W=\left(C^{*}-\bar{V}\right) \cup \cup\left(\partial O_{n}, d_{n}\right)$ and we take $g$ to be any cluster point of the sequence $\left\{g_{n}\right\}$, a normal family of functions. In each set $N\left(\partial O_{n}, d_{n}\right)$ the functions $g_{n+1}, g_{n+2}, g_{n+3}, \ldots$ are all analytic, and therefore $g$ is analytic in $W$. Also, $g$ is bounded by 1 on $W$, so $g$ is "admissible" for the compact set $\mathbf{C}^{*}-W$, when we extend $g$ to $\mathbf{C}^{*}$ by setting it equal to zero off $W$. Note that $\mathbf{C}^{*}-\bar{V} \subset W$ implies $\mathbf{C}^{*}-W \subset \bar{V}$, and since the set $S_{0}=\bigcup \partial O_{n}$ is contained in $W$, we have $\mathrm{C}^{*}-W \subset \bar{V}-S_{0}$. Thus $g$ is "admissible" for a compact subset of $\bar{V}-S_{0}$.

It is easy to see that $\|g-f\|_{C} \leqslant \epsilon / 2 R$, from which it follows that $\gamma(\bar{V})=$ $f^{\prime}(\infty) \leqslant\left|g^{\prime}(\infty)\right|+\epsilon \leqslant \gamma\left(\bar{V}-S_{0}\right)+\epsilon$. Since $\epsilon$ is arbitrary, the proof of Theorem 5 is complete.

One example worth looking at in the light of Theorem 5 is Example 3.11 of [20]. Here L. Zalcman describes a bounded open set $U$ with $\gamma(U) \neq \gamma(\bar{U})$. The set $U$ is a union of open discs with pairwise disjoint closures, and these discs cluster only on the line segment $L=[0,1]$. In this example, the diameters of the components of $U$, i.e., the diameters of the discs, converge to zero. Therefore $S_{0}=S$ and Theorem 5 tells us that $\gamma(\bar{U})=\gamma(\bar{U}-S)=\gamma(U \cup L)$. Thus the only reason that $\gamma(U) \neq \gamma(\bar{U})$ is that $\gamma(U) \neq \gamma(U \cup L)$, which is what we expected.

We wish now to describe some special classes of open sets.

DEFinition. Let $V$ be a bounded open set with components $\left\{V_{j}\right\}_{j=1}^{\infty}$. Suppose that the components of $V$ can be grouped together as

$$
\begin{aligned}
V_{1}, V_{2}, \ldots, V_{j_{1}} ; V_{j_{1}+1}, V_{j_{1}+2}, \ldots, V_{j_{2}} ; \ldots ; \\
V_{j_{n}+1}, V_{j_{n}+2}, \ldots, V_{j_{n+1}} ; \ldots
\end{aligned}
$$

and that this grouping has the property that, for each $n$, the sets $\bigcup_{j=j_{n}+1}^{j_{n+1}} V_{j}$ and $\bigcup_{j=j_{n+1}+1}^{\infty} V_{j}$ have disjoint closures. Then we will call $V$ a bounded open set of type I.

For a set $V$ of type I, the "disjoint closures" condition tells us that the 
set $\partial V-S$ is compact. That is, $S$ is relatively open in $\partial V$, hence $\partial V-S$ is relatively closed in $\partial V$, which says that $\partial V-S$ is a compact set in the plane. For arbitrary open sets $V, \partial V-S$ need not be closed. Probably the simplest example is the following. Let $\left\{D_{j}\right\}_{j=1}^{\infty}$ be a collection of open discs with pairwise disjoint closures, such that (i) $\bar{D}_{j} \subset D(0,1)$, (ii) $\bar{D}_{j}$ is contained in the upper half plane $\{z \in C$ : $\operatorname{Im} z>0\}$, and (iii) $\bigcup_{j=1}^{\infty} D_{j}$ clusters only on the set $\{0\} \cup\{1 / 2,1 / 3,1 / 4, \ldots\}$. Let $D_{0}=D(-i, 1)$ and let $V=\bigcup_{j=0}^{\infty} D_{j}$. Then $\partial V-S=\{1 / 2,1 / 3,1 / 4, \ldots\}$.

Definition. Let $V$ be bounded open set of type I. If $\partial V-S$ is a (compact) set of zero analytic capacity, then we call $V$ a bounded open set of type II. If, in addition, each component of $V$ is a square, then we call $V$ a bounded open set of type III. Now let $V$ be any bounded open set. If $\left(\partial V-S_{0}\right)$ is a set of zero analytic capacity, then we call $V$ a bounded open set of type $S_{0}$.

We have the inclusion relations III $\subset$ II $\subset$ I $\cap S_{0}$. Each of these inclusions is proper, and there is no inclusion relation between $I$ and $S_{0}$. The example just described is a set of type $S_{0}$ which is not of type $I$, and we will later describe a set of type I which is not of type $S_{0}$. A sequence of rectangles of width 1 , each contained in $D(0,2)$, clustering only on the unit interval, affords an example of a set which is of types I and $S_{0}$ but not of type II.

THEOREM 6. The following conjectures are equivalent.

(a) The two set functions $\alpha$ and $\gamma$ are semi-additive (for disjoint compact sets).

(b) $\gamma(V)=\gamma(\bar{V})$ for all bounded open sets of type $S_{0}$.

(c) $\gamma(V)=\gamma(\bar{V})$ for all bounded open sets of type II.

(d) $\gamma(V)=\gamma(\bar{V})$ for all bounded open sets of type III.

Proof. We first show $d \Rightarrow$ a. Assuming that $\gamma$ is not semi-additive, $A$. Davie [3, Theorem 5.2] constructs a compact set $E$ of zero analytic capacity, and a bounded set $F$, which is a countable union of closed, nonoverlapping squares, such that $E \cup F=\bar{F}$ and $\gamma(F)<\gamma(E \cup F)$. Let $V$ be the corresponding union of open squares. In the construction, Davie has disjoint compact sets $E_{n}$, $F_{n}$ at each stage, and each of these is a finite union of nonoverlapping closed squares. He then places $F_{n+1}-F_{n}$ in the interior of $E_{n}$ (and also places $E_{n+1}$ in the interior of $E_{n}$ ) and he later defines $F=\bigcup_{n=1}^{\infty} F_{n}$. We may then take the groupings in the definition of sets of type $I$ to be: squares of $F_{1}$; squares of $\left(F_{2}-F_{1}\right)$; squares of $\left(F_{3}-F_{2}\right) ; \ldots$ Thus $V$ is a set of type $\mathrm{I}$, and since the set $\partial V-S$ is equal to $E$, and each component of $V$ is a square, $V$ is also a set of type III. Finally $\gamma(V)=\gamma(V \cup S)=\gamma(F)<\gamma(E \cup F)=\gamma(\bar{V})$. This shows that $d$ implies the semi-additivity of $\gamma$, and then $d$ implies a, because the semiadditivity of $\gamma$ is equivalent to that of $\alpha$. 
Now assume that $\gamma$ is semi-additive. Let $V$ be a set of type $S_{0}$ and let $A=\partial V-S_{0}$, a set of zero analytic capacity. We must have $\gamma(V)=\gamma(E \cup V)$ for all compact sets $E$ of zero analytic capacity, hence for all compact sets $E \subset$ $A$. We claim that this implies $\gamma(V)=\gamma(A \cup V)$, from which it will follow that $\gamma(V)=\gamma(A \cup V)=\gamma\left(\bar{V}-S_{0}\right)=\gamma(\bar{V})$ by Theorem 5 .

Let $L \subset A \cup V$ be compact. Then $L \subset V \cup(L \cap \partial V)$. Since $L \cap \partial V$ is a compact subset of $A$, we have $\gamma(L) \leqslant \gamma(V \cup(L \cap \partial V))=\gamma(V)$. Taking the supremum over $L$ we see that $\gamma(A \cup V) \leqslant \gamma(V) \leqslant \gamma(A \cup V)$. This proves a $\Rightarrow \mathrm{b}$, and the final implications, $\mathrm{b} \Rightarrow \mathrm{c} \Rightarrow \mathrm{d}$, are trivial.

3. Examples. For a set $V$ of type II, $\partial V-S$ is a compact set of zero analytic capacity. For a set $V$ of type $S_{0}, \partial V-S_{0}$ has zero analytic capacity, but does that mean that $\left(\partial V-S_{0}\right)^{-}$is a compact set of zero capacity? Our first example shows that this need not be true. Let $V_{1}$ be the square $(0,1) \times$ $(-1,0)$. Let $\left\{r_{m}\right\}_{m=1}^{\infty}$ be an enumeration of the rationals in $[0,1]$. Let $L_{0}$ be the line $y=2$ in the $x y$-plane, and for each positive integer $n$, let $L_{n}$ be the line $y=1 / n$. For each pair of positive integers $m$ and $n$, with $m \leqslant n$, let $z_{n, m}$ be the point $\left(r_{m}, 1 / n\right)$. See the figure. Let $\left\{D_{n, m, j}\right\}_{j=1}^{\infty}$ be a sequence of discs with pairwise disjoint closures contained in the open strip between $L_{n-1}$ and $L_{n}$ such that (i) these discs cluster only at the point $z_{n, m}$ and (ii) $D_{n, m, j}$ has radius less than $2^{-n-m-j-3}$. We can do this for each pair of positive integers $m$ and $n$ with $m \leqslant n$, and we can insure that all discs $D_{n, m, j}$ have pairwise disjoint closures. Let $V$ be the set $V=\bigcup_{n=1}^{\infty} \bigcup_{m=1}^{n} \bigcup_{j=1}^{\infty} D_{n, m, j} \cup V_{1}$.

Then we have $S=S_{0}$, and $\partial V-S=\left\{z_{n, m}: m=1, \ldots, n ; n=1,2, \ldots\right\}$. Since $\partial V-S$ is countable it has zero analytic capacity. However its closure contains the inierval $[0,1]$ and has capacity $1 / 4$. In this particular example we do have $\gamma(V)=\gamma(\bar{V})$ via Theorem $4 c$, because only countably many boundary points of $V$ fail to belong to the set $S$.

If we replace $V$ by $U=V-V_{1}$ then the discs of $U$ still cluster on $[0,1]$. Estimating $\gamma(U)$ by perimeter $/ 2 \pi$ according to Proposition 3.8 of [20], we see that $\gamma(U) \leqslant 1 / 8<1 / 4=\gamma([0,1]) \leqslant \gamma(\bar{U})$. The set $U$ is no longer of type $S_{0}$ and so the fact that $\gamma(U) \neq \gamma(\bar{U})$ is not disturbing.

Now we would like to look at some examples of sets $V$ of types I and II. Sets of type I include Zalcman's Example 3.11 and the set $U$ just described, so we cannot hope to have $\gamma(V)=\gamma(\bar{V})$ for all such sets. The first set we describe will be a set of type I which is not of type II or of type $S_{0}$. All five parts of Theorem 1 will fail for this set, and we will not have $\gamma(V)=\gamma(\bar{V})$. The major example of this section will be a set of type II for which all five parts of Theorem 1 are true, and therefore $\gamma(V)=\gamma(\bar{V})$. However if we were to attempt to show $\gamma(V)=\gamma(\bar{V})$ for this set by using Corollary 1 instead of Theorem 1, we would not be able to do so. The lim inf condition of Corollary 1 is not precise 
$L_{0}: y=2$
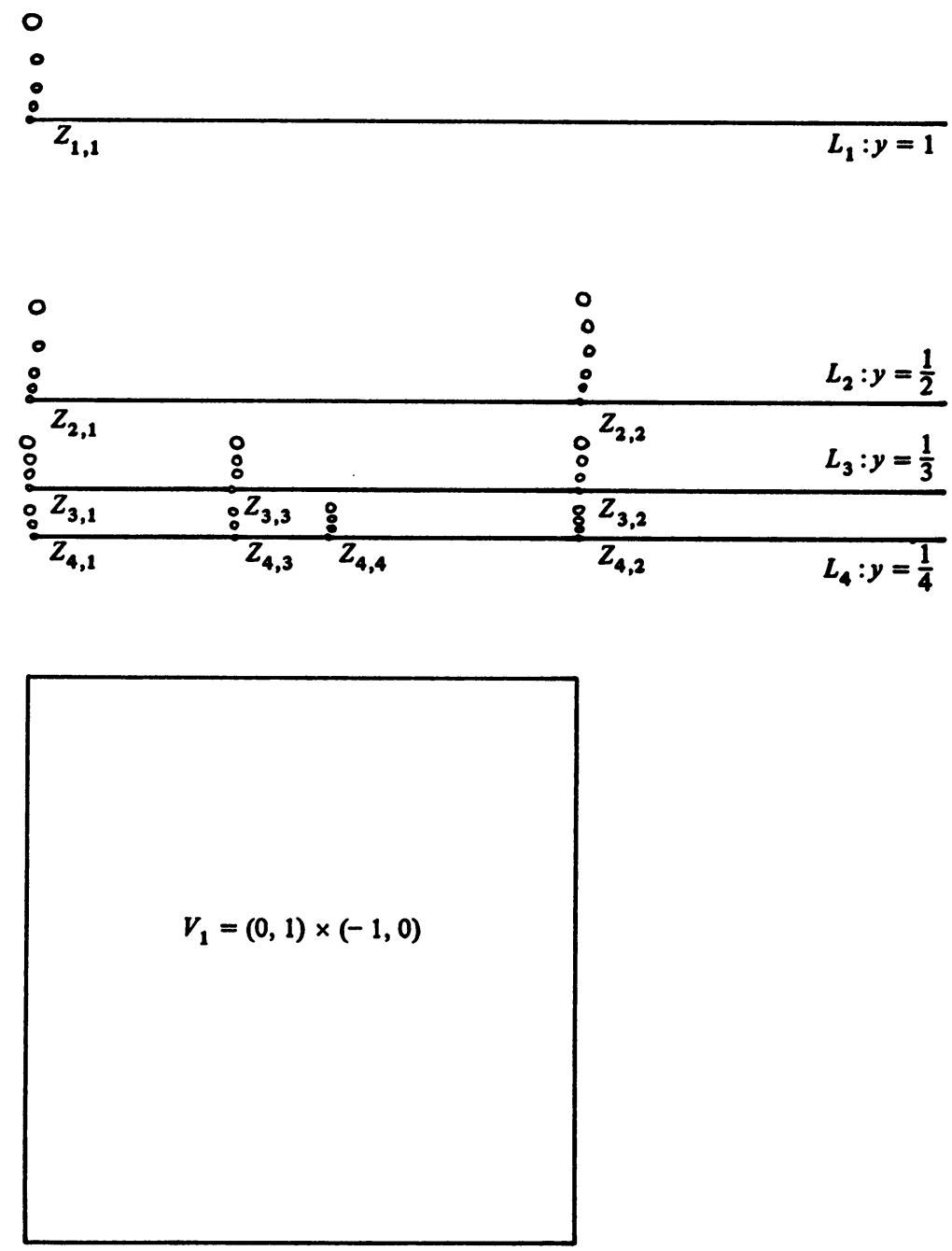

enough. We will also describe a set of type II for which we do not know whether $\gamma(V)=\gamma(\bar{V})$. We will be able to relate $\alpha(V)$ and $\alpha(\bar{V})$, though. Each of the open 
sets to be described will have $S=S_{0}$ because the diameters of the components will converge to zero.

Let $E=\bigcap_{n=0}^{\infty} E_{n}$, where $E_{0}$ is the closed unit square, $E_{n}$ consists of $4^{n}$ closed squares of side $3^{-n}$, and each component of $E_{n}$ contains four components of $E_{n+1}$, these being the four corner squares of side $3^{-n-1}$. The components of $E_{n}$ will be indexed as $E_{n, j}\left(1 \leqslant j \leqslant 4^{n}\right)$ and we take $z_{n, j}$ to be the center of $E_{n, j}$. Let $0<r_{n} \leqslant 1 / 6 \cdot 3^{n}$ and let $D_{n, j}=D\left(z_{n, j}, r_{n}\right)$. If we take $V$ to be the union of all $D_{n, j}$ then $V$ is a bounded open set of type I. The set $E$ is the product of the usual Cantor set with itself, and according to $[18], \gamma(E) \geqslant \alpha(E)>0$. So $V$ is not a set of type II or of type $S_{0}$. If the $r_{n}$ are chosen very small, we find, as in Zalcman's example, that $\gamma(V) \leqslant \Sigma 4^{n} r_{n}<\gamma(E) \leqslant \gamma(\bar{V})$. This tells us that part e of Theorem 1 fails for $W=\mathrm{C}^{*}-\bar{V}$ and our set $E$. So all parts of that theorem fail, and there would be no hope in looking at the ratio of capacities. On the other hand, if we take $r_{n}=1 / 6 \cdot 3^{n}$, then the discs would shrink at the same rate as the squares $E_{n, j}$, and we would get a capacity estimate giving $\gamma(V)=\gamma(\bar{V})$. (For $z \in E$ and for any positive $\delta$, we can always construct an arc in $D(z, \delta) \cap V$ whose diameter, divided by $\delta$, stays away from zero.)

Now let us keep the same notation, but use it in a different situation. We take the squares of $E_{n}$ to have side $5^{-n}$ and we take $0<r_{n} \leqslant 5^{-n-1}$. We again get a set $V$ of type I, but this time $V$ is also of type II. To see that, we estimate $\gamma\left(E_{n}\right)$ by means of Proposition 3.8 of [20]. We have perimeter of $E_{n}=4^{n} \cdot 4 \cdot 5^{-n}$, so perimeter of $E_{n} / 2 \pi \leqslant(4 / 5)^{n}$. Since $E \subset E_{n}$ for all $n$, $\gamma(E) \leqslant$ perimeter of $E / 2 \pi=0$. Thus $V$ is of type II, and we hope that $\gamma(V)=$ $\gamma(\bar{V})$. There are two ways in which we can show that this is so. The first method modifies the proof of Theorem 3.1 of [7] to treat pointwise bounded approximation instead of uniform approximation. This is the sort of thing we have been doing in $\$ 1$ and it does apply to Garnett's proof, since our set $E$ is his set $E_{0.6}$. The second method is the estimation of capacities. This depends on knowing the $r_{n}$. We will give an example of these estimates, because it will show a situation in which Corollary 1 would fail to provide any information, while Theorem 1 is successful.

We take $r_{n}=25^{-n-1}$, and we claim that for each $z \in E$,

$$
\liminf _{\delta \rightarrow 0} \frac{\gamma(D(z, \delta) \cap V)}{\gamma(D(z, \delta) \cap \bar{V})}>0
$$

but

$$
\lim _{\delta \rightarrow 0} \frac{\gamma(D(z, \delta) \cap(V \cup S))}{\delta}=0 .
$$

Choose $\delta$ with $0<\delta<1 / 10$ and fix $z \in E$. Then there is a positive integer $N$ such that $D(z, \delta)$ contains a square of $E_{N}$ but does not contain any square of $E_{N-1}$. This tells us that $N$ and $\delta$ are related by $\sqrt{2} / 5^{N}<2 \delta$ and 
$\delta<\sqrt{2} / 5^{N-1}=5 \sqrt{2} / 5^{N}$. That is,

$$
\sqrt{2} / 2 \cdot 5^{-N}<\delta<5 \sqrt{2} \cdot 5^{-N}
$$

Let $Q$ be the square of $E_{N}$ which contains $z$. Since $D(z, \delta)$ contains some square (or squares) of $E_{N}$, it follows that $Q \subset D(z, \delta)$. Next let $Q^{\prime}$ be the square of $E_{N-1}$ which contains $Q$. The distance from $z \in Q^{\prime}$ to any other square of $E_{N-1}$ is at least $3 / 5^{N-1}>\delta$. Therefore $D(z, \delta)$ meets no square of $E_{N-1}$ other than $Q^{\prime}$, and

$$
\begin{gathered}
D(z, \delta) \cap(V \cup S) \cap E_{N-1}=D(z, \delta) \cap(V \cup S) \cap Q^{\prime}, \\
D(z, \delta) \cap \bar{V} \cap E_{N-1}=D(z, \delta) \cap \bar{V} \cap Q^{\prime} .
\end{gathered}
$$

Let $A=\bigcup_{m=0}^{N-2} \bigcup_{k=1}^{4 m} \bar{D}_{m, k}$. Among those discs which make up $A$, it is possible for only one of them to meet $D(z, \delta)$. Such a disc, if there is one, will be $\bar{D}_{N-2, j}$ for some $j$, and this will be the disc nearest to the square $Q^{\prime}$ of $E_{N-1}$. That is, it will be the disc with the same center as $Q^{\prime \prime}$, the square of $E_{N-2}$ which contains $Q^{\prime}$. (Note that (1) implies $\sqrt{2} / 2 \cdot 5^{N}<\delta<1 / 10$, or $5 \sqrt{2}<5^{N}$, or $2 \leqslant N$. Thus $N-2$ as a subscript is meaningful.) Therefore

$$
\begin{gathered}
D(z, \delta) \cap(V \cup S) \cap A \subset D(z, \delta) \cap(V \cup S) \cap \bar{D}_{N-2, j} \subset \bar{D}_{N-2, j} \\
D(z, \delta) \cap \bar{V} \cap A \subset D(z, \delta) \cap \bar{V} \cap \bar{D}_{N-2, j} \subset \bar{D}_{N-2, j}
\end{gathered}
$$

Since $V \cup S \subset \bar{V} \subset E_{N-1} \cup A$ we find from (2) through (5) that

$$
\begin{gathered}
D(z, \delta) \cap(V \cup S) \subset\left[D(z, \delta) \cap(V \cup S) \cap Q^{\prime}\right] \cup \bar{D}_{N-2, j^{\prime}} \\
D(z, \delta) \cap \bar{V} \subset\left[D(z, \delta) \cap \bar{V} \cap Q^{\prime}\right] \cup \bar{D}_{N-2, j^{*}}
\end{gathered}
$$

We estimate the capacities of these sets by perimeter $2 \pi$.

From (6) we see that $D(z, \delta) \cap(V \cup S)$ is contained in the union of the following closed discs:

one disc, $\bar{D}_{N-2, j}$, of radius $25^{-N+1}$;

one disc, say $\bar{D}_{N-1,1}^{\prime}$, of radius $25^{-N}$;

four discs, say $\bar{D}_{N, 1}^{\prime}, \ldots, \bar{D}_{N, 4}^{\prime}$, each of radius $25^{-N-1}$;

16 discs, say $\bar{D}_{N+1,1}^{\prime}, \ldots, \bar{D}_{N+1,16}^{\prime}$, each of radius $25^{-N-2}$; etc.

Note that each disc $D_{N+m, k}^{\prime}$ is one of the $D_{N+m, l}$, for $m=-1,0,1,2$, $\ldots ; k=1,2, \ldots, 4^{m+1} ; l=1,2, \ldots, 4^{N+m}$. Adding the radii, we get $25^{-N}(25+25 / 21)<50 / 25^{N}$. Thus

$$
\gamma(D(z, \delta) \cap(V \cup S))<100\left(\sqrt{2} / 2 \cdot 5^{N}\right)^{2}<100 \delta^{2} .
$$

In the same way, $D(z, \delta) \cap \bar{V}$ is contained in the union of: one disc, $\bar{D}_{N-2, j}$; one disc, $\bar{D}_{N-1,1}^{\prime} ;$ four discs $\bar{D}_{N, 1}^{\prime}, \ldots, \bar{D}_{N, 4}^{\prime} ;$ etc. $4^{n-1}$ discs, $\bar{D}_{N+n-2,1}^{\prime}, \ldots, \bar{D}_{N+n-2,4^{n-1}}^{\prime}$; and $4^{n}$ squares, $E_{N+n-1, k}\left(1 \leqslant k \leqslant 4^{n}\right)$, 
where $n$ is any positive integer whatsoever. Then $\gamma\left(D(z, \delta) \cap \bar{V} \leqslant 50 / 25^{N}+4^{n}\right.$ $\cdot 4 \cdot 5^{-N-n+1} / 2 \pi \leqslant 100 \delta^{2}+4 \cdot 5^{-N}(4 / 5)^{n}$. We will choose $n \geqslant 8 N$, because $(4 / 5)^{8}<1 / 5$, and so $(4 / 5)^{n} \leqslant(4 / 5)^{8 N} \leqslant(1 / 5)^{N}$. Therefore

$$
\gamma(D(z, \delta) \cap \bar{V}) \leqslant 100 \delta^{2}+4 \cdot 25^{-N} \leqslant 108 \delta^{2} .
$$

One more estimate we need is a lower bound for $\gamma(D(z, \delta) \cap V)$. Since $D(z, \delta)$ contains a square of $E_{N}, D(z, \delta) \cap V$ contains the disc concentric with this square. The radius of the disc is $25^{-N-1}=(25 \cdot 50)^{-1}\left(50 \cdot 25^{-N}\right) \geqslant$ $(1250)^{-1} \delta^{2}$. Therefore

$$
\gamma(D(z, \delta) \cap V) \geqslant \delta^{2} / 1250 \text {. }
$$

From (9) and (10) we have, for $z \in E$ and $0<\delta<1 / 10, \gamma(D(z, \delta) \cap V) /$ $\gamma\left(D(z, \delta) \cap \bar{V} \geqslant(1250 \cdot 108)^{-1} \geqslant 10^{-6}\right.$, so that

$$
\liminf _{\delta \rightarrow 0} \frac{\gamma(D(z, \delta) \cap V)}{\gamma(D(z, \delta) \cap \bar{V})} \geqslant 10^{-6}>0 .
$$

However, as $\delta$ goes to zero, $0 \leqslant \lim \inf \gamma(D(z, \delta) \cap(V \cup S)) / \delta \leqslant$ $\lim \sup \gamma(D(z, \delta) \cap(V \cup S)) / \delta \leqslant \lim \sup 100 \delta=0$. Theorem 1 , with $W=$ $\mathrm{C}^{*}-\bar{V}$, and with $E$ (of Theorem 1) $=\partial V=E \cup S$, shows that $\gamma(V)=\gamma(\bar{V})$.

The short form, Corollary 1 , gives no information at all, even if we take $E$ (in Corollary 1) to be the Cantor set $E$ instead of the larger set, $\partial V$.

One more Cantor set is the one in which $E_{n}$ consists of squares of side $4^{-n}$. If we take $0<r_{n} \leqslant 4^{-n-1}$, then $V$ is a set of type I. Proving that $\gamma(E)=$ 0 , and hence that $V$ is a set of type II, is very difficult, but John Garnett has shown in [8] that this is so. So again we hope that $\gamma(V)=\gamma(\bar{V})$. If $r_{n}=4^{-n-1}$, then the discs shrink at the same rate as the squares, and by producing arcs we can show $\lim$ inf $\gamma(D(z, \delta) \cap V) / \delta>0$, which means that $\gamma(V)=\gamma(\bar{V})$. However if $r_{n}$ is much smaller, for instance $r_{n}=16^{-n-1}$, then we do not know whether $\gamma(V)=\gamma(\bar{V})$. We will show that $\alpha(\bar{V})<1.34 \alpha(V)$ in this case.

Fix $a, b$ with $0<a<1 / 4,0<b<1 / 4$. Let $E$ be the Cantor set just described and let $V$ be the open set corresponding to any choice of $r_{n}$ such that $0<r_{n} \leqslant$ $a b^{n}$. Let $f$ be a function $C$-admissible for the set $\bar{V}$. For any $n, f^{\prime}(\infty)$ is the Cauchy integral of $f$ around the boundaries of the sets $D_{m, k}, E_{n, j}$ where $1 \leqslant k$ $\leqslant 4^{m}, 0 \leqslant m \leqslant n-1,1 \leqslant j \leqslant 4^{n}$. We wish first to examine the integral around the $E_{n, j}$. We apply Theorem III.1.1 of [19] with $g=$ interior of $E_{n, j}$ and $e=$ $E_{n, j}$. Let $\omega(\cdot)$ be the modulus of continuity of $f$, and let $\lambda$ be the constant of Theorem III.1.1 for a square. ( $\lambda$ is an absolute constant; see the paragraph following the statement of Theorem III.1.1). Then

$$
\left|\int_{\partial E_{n, j}} f(z) d z\right| \leqslant \lambda \cdot \omega(\alpha) \cdot \alpha
$$


where

$$
\alpha=\alpha\left(\text { interior } E_{n, j}\right) \leqslant \text { side of } E_{n, j}=4^{-n} \text {. }
$$

Therefore

$$
\sum_{j=1}^{4^{n}}\left|\frac{1}{2 \pi i} \int_{\partial E_{n, j}} f(z) d z\right| \leqslant \lambda \cdot \omega\left(4^{-n}\right) \rightarrow 0 \quad \text { as } n \rightarrow \infty .
$$

From this we see that

$$
\begin{aligned}
f^{\prime}(\infty) & =\sum_{m=0}^{n-1} \sum_{k=1}^{4^{m}} \frac{1}{2 \pi i} \int_{\partial D_{m, k}} f(z) d z \\
& +\sum_{j=1}^{4^{n}} \frac{1}{2 \pi i} \int_{\partial E_{n, j}} f(z) d z \\
& \rightarrow \frac{1}{2 \pi i} \int_{S} f(z) d z+0
\end{aligned}
$$

Now

$$
\begin{aligned}
\sum_{m=0}^{\infty} \sum_{k=1}^{4^{m}}\left|\frac{1}{2 \pi i} \int_{\partial D_{m, k}} f(z) d z\right| & \leqslant \sum_{m=0}^{\infty} \sum_{k=1}^{4^{m}}(2 \pi)^{-1} \cdot 1 \cdot\left(2 \pi r_{m}\right) \\
& \leqslant \sum_{m=0}^{\infty} a(4 b)^{m}=a /(1-4 b) .
\end{aligned}
$$

Thus

$$
\alpha(\bar{V})=\sup \left|f^{\prime}(\infty)\right|=\sup \left|(2 \pi i)^{-1} \int_{S} f(z) d z\right| \leqslant a /(1-4 b) .
$$

But $\alpha(V) \geqslant \alpha\left(D_{0,1}\right)=r_{0}$ so

$$
\alpha(\bar{V}) \leqslant \alpha(V) \cdot a / r_{0} \cdot 1 /(1-4 b) .
$$

In the case that $r_{n}=16^{-n-1}$, we have $a=b=r_{0}=1 / 16$, and $\alpha(\bar{V}) \leqslant 4 / 3 \cdot \alpha(V)$. The inequality (12) is not very useful for $b$ near $1 / 4$. Another way of comparing $\alpha(V)$ and $\alpha(\bar{V})$ is: $\alpha(\bar{V}) \leqslant \alpha\left(E_{0}\right)<0.6 ; \alpha(V) \geqslant \alpha\left(D_{0,1}\right)=r_{0}$; and

$$
\alpha(\bar{V})<\alpha(V) \cdot 3 / 5 r_{0}
$$

If it were possible to remove the requirement of continuity in (11), we would find that (12) holds with $\alpha$ replaced by $\gamma$. For let $L \subset \bar{V}-S$ be compact and let $f$ be admissible for $L$. The integral of $f$ on $S$ makes sense because $f$ is continuous on $S$. In fact $f$ is analytic on a neighborhood of $S$. Then $\left|f^{\prime}(\infty)\right| \leqslant$ $a /(1-4 b)$. Taking the supremum over $f$ yields $\gamma(L) \leqslant a /(1-4 b)$. Taking the supremum over $L$ yields $\gamma(\bar{V})=\gamma(\bar{V}-S) \leqslant a /(1-4 b)$, according to Theorem 5 , and so we proceed as before. Of course (13) does not require continuity of $f$ on $\mathbf{C}$, and so (13) does hold with $\gamma$ replacing $\alpha$. 


\section{BIBLIOGRAPHY}

1. Andrew Browder, Introduction to function algebras, Benjamin, New York, 1969. MR 39 \#7431.

2. Philip C. Curtis, Jr., Peak points for algebras of analytic functions, J. Functional Analysis 3 (1969), 35-47. MR 39 \#463.

3. Alexander M. Davie, Analytic capacity and approximation problems, Trans. Amer. Math. Soc. 171 (1972), 409-444. 1969.

4. Theodore W. Gamelin, Uniform algebras, Prentice-Hall, Englewood Cliffs, N. J.,

5. T. W. Gamelin and J. Garnett, Pointwise bounded approximation and hypodirichlet algebras, Bull. Amer. Math. Soc. 77 (1971), 137-141. MR 42 \#6491.

6. - Pointwise bounded approximation and Dirichlet algebras, J. Functional Analysis 8 (1971), 360-404. MR 46 \#4153.

7. John Garnett, Metric conditions for rational approximation, Duke Math. J. 37 (1970), 689-699.

8. Positive length but zero analytic capacity, Proc. Amer. Math. Soc. 24 (1970), 696-699. MR 42 \#4746.

9. - Analytic capacity and measure, Lecture Notes in Math., vol. 297, SpringerVerlag, Berlin and New York, 1972.

10. N. S. Landkof, Foundations of modern potential theory, "Nauka", Moscow, 1966; English transl., Die Grundlehren der math. Wissenschaften, Band 180, SpringerVerlag, Berlin and New York, 1972. The table on p. 172 gives values for the logarithmic capacity of several sets in the plane, and these are also the values of the analytic capacity whenever the sets are compact and connected. MR 35 \#5644.

11. Trevor J. McMinn, Linear measures of some sets of the Cantor type, Proc. Cam. bridge Philos. Soc. 53 (1957), 312-317. MR 19, 20.

12. Christian Pommerenke, Über die analytische Kapazität, Arch. Math. 11 (1960), 270-277. MR 22 \#5740c.

13. John F. Randolph, Some properties of sets of the Cantor type, J. London Math. Soc. 16 (1941), 38-42. MR 3, 226.

14. Walter Rudin, Real and complex analysis, McGraw-Hill, New York, 1966, 1974. MR 35 \#1420; 49 \#8783.

15. N. A. Sirokov, A certain property of analytic capacity, Vestnik Leningrad. Univ. 1971, no. 19, 75-82. (Russian) MR 46 \#2034.

16. Certain properties of analytic capacity, Vestnik Leningrad. Univ. 1972, no. 19, 77-86. (Russian) MR 46 \#345.

17. N. A. Šrokov, Analytic capacity of sets that are close to a smooth curve, Vestnik Leningrad. Univ. No. 19 Mat. Meh. Astronom. Vyp. 4 (1973), 73-78, 153. (Russian) MR 48 \#11486. 144-150.

18. P. Urysohn, Sur une fonction analytique partout continue, Fund. Math. 4 (1923),

19. A. G. Vituškin, Analytic capacity of sets in problems of approximation theory, Uspehi Mat. Nauk 22 (1967), no. 6 (138), $141-199=$ Russian Math. Surveys 22 (1967), no. 6, 139-200. MR 37 \#404.

20. Lawrence Zalcman, Analytic capacity and rational approximation, Lecture Notes in Math., no. 50, Springer-Verlag, Berlin and New York, 1968. MR 37 \#3018. 